\title{
A NOTE ON MULTIFLOW LOCKING THEOREM
}

\author{
Hiroshi Hirai \\ Research Institute for Mathematical Sciences, \\ Kyoto University
}

(Received November 27, 2009; Revised February 17, 2010)

\begin{abstract}
This note addresses the undirected multiflow (multicommodity flow) theory. A multiflow in a network with terminal set $T$ can be regarded as a single commodity $(A, T \backslash A)$-flow for any nonempty proper subset $A \subset T$ by ignoring flows not connecting $A$ and $T \backslash A$. A set system $\mathcal{A}$ on $T$ is said to be lockable if for every network having $T$ as terminal set there exists a multiflow being simultaneously a maximum $(A, T \backslash A)$-flow for every $A \in \mathcal{A}$. The multiflow locking theorem, due to Karzanov and Lomonosov, says that $\mathcal{A}$ is lockable if and only if it is 3 -cross-free.

A multiflow can also be regarded as a single commodity $(A, B)$-flow for every partial cut $(A, B)$ of terminals, where a partial cut is a pair of disjoint subsets (not necessarily a bipartition). Based on this observation, we study the locking property for partial cuts, and prove an analogous characterization for a lockable family of partial cuts.
\end{abstract}

Keywords: Network flow, multiflow locking theorem, 3-cross-free family

\section{Introduction}

By a network $(G, T, c)$ we mean a triple of an undirected graph $G=(V G, E G)$, a specified node subset $T \subseteq V G$, and a nonnegative edge-capacity $c: E G \rightarrow \mathbf{R}_{+}$. A node in $T$ is called a terminal, and a node in $V G \backslash T$ is called an inner node. A multiflow (multicommodity flow) is a pair $f=(\mathcal{P}, \lambda)$ of paths $\mathcal{P}$ connecting distinct terminals and a nonnegative flow-value function $\lambda: \mathcal{P} \rightarrow \mathbf{R}_{+}$satisfying the capacity constraint $\sum_{P \in \mathcal{P}: e \in P} \lambda(P) \leq c(e)$ for each edge $e \in E G$. A multiflow is said to be integral if its flow-value function is integer-valued. For a nonempty proper subset $A \subset T$, any multiflow $f$ can be regarded as a single commodity $(A, T \backslash A)$-flow by ignoring paths not connecting $A$ and $T \backslash A$. One of interesting phenomena in multiflows is: for a special set system $\mathcal{A} \subseteq 2^{T} \backslash\{\emptyset, T\}$ there always exists a multiflow $f$ being simultaneously a maximum $(A, T \backslash A)$-flow for all $A \in \mathcal{A}$. For example, take $\mathcal{A}$ as the set of all singletons $\{\{s\} \mid s \in T\}$. Then Lovász [16] and Cherkassky [2] independently showed that there exists a multiflow being simultaneously a maximum $(s, T \backslash s)$-flow for all $s \in T$. Moreover, if the capacity $c$ is integer-valued, then there exists a half-integral multiflow of this property.

For a multiflow $f$, we say " $f$ locks $\mathcal{A}$ " if $f$ is a maximum $(A, T \backslash A)$-flow for all $A \in \mathcal{A}$. Then $\mathcal{A}$ is said to be lockable if for every network $(G, T, c)$ there exists a multiflow locking $\mathcal{A}$. The multiflow locking theorem, due to Karzanov and Lomonosov [14], gives a complete characterization of such a lockable set system. Two subsets $A, B \subseteq T$ are said to be $c r o s s i n g$ if none of $A \backslash B, B \backslash A, A \cap B$, and $T \backslash(A \cup B)$ is empty. A network $(G, T, c)$ is said to be inner Eulerian if $c$ is integer-valued and every inner node has even degree.

Theorem 1.1 ([14]). Let $\mathcal{A} \subseteq 2^{T} \backslash\{\emptyset, T\}$ be a set system on $T$. Then $\mathcal{A}$ is lockable if 
and only if $\mathcal{A}$ has no pairwise crossing triple. In addition, if lockable, then there exists an integral multiflow locking $\mathcal{A}$ in every inner Eulerian network $(G, T, c)$.

See $[7,15]$ for proofs, and also see $[12]$ and $[18,73.3 \mathrm{c}]$ for further information. The aim of this note is to give an extension of this result. A pair $(A, B)$ of two disjoint nonempty subsets is called a partial cut; we do not distinguish $(A, B)$ and $(B, A)$. For a partial cut $(A, B)$ on $T$, any multiflow $f$ can also be regarded as a single commodity $(A, B)$-flow (by ignoring paths not connecting $A$ and $B$ ). So we can extend the locking concept for partial cuts. Let $\mathcal{A}$ be a set of partial cuts on $T$. For a multiflow $f$, we say " $f$ locks $\mathcal{A}$ " if $f$ is a maximum $(A, B)$-flow for all $(A, B) \in \mathcal{A}$. Then $\mathcal{A}$ is said to be lockable if there exists a multiflow locking $\mathcal{A}$ in every network $(G, T, c)$.

Our main result is an analogous characterization of a lockable system of partial cuts. Two partial cuts $(A, B)$ and $(C, D)$ are said to be laminar if one of the following four cases holds: (i) $A \subseteq C, B \supseteq D$, (ii) $A \subseteq D, B \supseteq C$, (iii) $A \supseteq C, B \subseteq D$, (iv) $A \supseteq D, B \subseteq C$. Otherwise $(A, B)$ and $(C, D)$ are said to be crossing, and, in addition, said to be regularly crossing if $A \cup B=C \cup D$ and irregularly crossing if $A \cup B \neq C \cup D$. A terminal $s$ is said to be proper if $s \in A \cup B$ for all $(A, B) \in \mathcal{A}$, and improper otherwise. A network $(G, T, c)$ is said to be properly inner Eulerian (with respect to $\mathcal{A}$ ) if it is inner Eulerian and each improper terminal has even degree.

Theorem 1.2. Let $\mathcal{A}$ be a set of partial cuts on $T$. Then $\mathcal{A}$ is lockable if and only if $\mathcal{A}$ has no pairwise crossing triple and no irregularly crossing pair. In addition, if lockable, then there exists an integral multiflow locking $\mathcal{A}$ in every properly inner Eulerian network $(G, T, c)$.

This theorem includes the previous one for a special case where $A \cup B=T^{\prime} \subseteq T$ for each $(A, B) \in \mathcal{A}$. Originally we found this result by using a framework in $[10,11]$; we can associate $\mathcal{A}$ with a cubital folder complex $\mathcal{K}_{\mathcal{A}}$, and can derive Theorem 1.2 by $[11$, Proposition 2.9 , Theorem 5.1]. However, we here prove it by a basic technique involving splitting-off and submodularity of cuts, similar to that in $[7,16]$.

\section{Proof}

Let $(G, T, c)$ be a network, possibly having multiple edges and loops. An edge $e$ joining nodes $x$ and $y$ is denoted by $x y$. We begin to prove the only-if part.

Only-if part. Let $\mathcal{A}$ be a set of partial cut on $T$. Suppose first that $\mathcal{A}$ has an irregularly crossing pair $(A, B),(C, D)$. We may assume $A \backslash(C \cup D) \neq \emptyset$. Take $s \in A \backslash(C \cup D)$. Suppose the case where both $B \cap C$ and $B \cap D$ are nonempty. Take $t \in B \cap C$ and $u \in B \cap D$. Consider the network on $T$ consisting of only two edges $s t, t u$ with unit capacity. Then there is no multiflow locking $\{(A, B),(C, D)\}$. Indeed, to lock $(C, D)$, we need to push unit $(t, u)$-flow passing through $t, s, u$ in order. However if push, then it is impossible to add flows to lock $(A, B)$. Next suppose the case where $B \backslash(C \cup D)$ is nonempty. We may assume that $A \cap C$ or $A \cap D$ is empty; otherwise it reduces to the case above. Take $s^{\prime} \in B \backslash(C \cup D)$, $t \in C$, and $t^{\prime} \in D$. Consider the network consisting of four edges $s t, t s^{\prime}, s^{\prime} t^{\prime}, t^{\prime} s$ with unit capacity. Again there is no multiflow locking $\{(A, B),(C, D)\}$. Indeed, we may assume $t^{\prime} \notin A$. To lock $(A, B)$, we need to push unit $\left(s, s^{\prime}\right)$-flow passing through $s, t^{\prime}, s^{\prime}$ in order. If push, then it is impossible to lock $(C, D)$. So we may assume $B \subseteq C$. Since $(A, B)$ and 
$(C, D)$ are not laminar, $D \backslash A$ is nonempty. Take $t \in B \subseteq C$ and $u \in D \backslash A$. Consider the network consisting of two edges $s t, s u$ of unit capacity. Again there is no multiflow locking $\{(A, B),(C, D)\}$.

So we may assume that $\mathcal{A}$ has no irregularly crossing pair and has pairwise crossing triple $\left(A_{i}, B_{i}\right)(i=1,2,3)$. Then $A_{1} \cup B_{1}=A_{2} \cup B_{2}=A_{3} \cup B_{3}$. In this case, we can directly use a proof $[15$, p. 44] of the only-if part of the ordinary locking theorem (Theorem 1.1).

If part. We need some notions. For a partial cut $(X, Y)$, the capacity $c(X, Y)$ is the sum of capacity of edges $e$ joining $X$ and $Y$. A cut is a bipartition $(X, Y)$ of node set $V G$. For a partial cut $(A, B)$ on $T$, a cut $(X, Y)$ is called an $(A, B)$-cut if $A \subseteq X$ and $B \subseteq Y$. The minimum capacity of $(A, B)$-cuts is denoted by $\kappa_{A, B}$. The cut distance $\delta_{A, B}: T \times T \rightarrow \mathbf{R}_{+}$ is defined by

$$
\delta_{A, B}(s, t)= \begin{cases}1 & \text { if }(s, t) \in A \times B \text { or }(t, s) \in A \times B, \quad(s, t \in T) . \\ 0 & \text { otherwise }\end{cases}
$$

Let $\mathcal{A}$ be a set of partial cuts on $T$. For a nonnegative weight $\alpha: \mathcal{A} \rightarrow \mathbf{R}_{+}$, let $\mu_{\mathcal{A}, \alpha}=$ $\sum_{(A, B) \in \mathcal{A}} \alpha(A, B) \delta_{A, B}$. Consider the following maximum multiflow problem:

$$
\operatorname{Max} . \sum_{P \in \mathcal{P}} \mu_{\mathcal{A}, \alpha}\left(s_{P}, t_{P}\right) \lambda(P) \text { s.t. } f=(\mathcal{P}, \lambda): \text { multiflow in }(G, T, c) .
$$

Here $s_{P}, t_{P}$ denote the ends of $P$. For a multiflow $f$, let $\operatorname{val}_{\mathcal{A}, \alpha}(f)$ denote the objective value of (2.1). Then we have

$$
\operatorname{val}_{\mathcal{A}, \alpha}(f) \leq \sum_{(A, B) \in \mathcal{A}} \alpha(A, B) \kappa_{A, B}
$$

Indeed, this follows from

$$
\sum_{P \in \mathcal{P}} \delta_{A, B}\left(s_{P}, t_{P}\right) \lambda(P)=(\text { the total flow-value of }(A, B) \text {-flows in } f) \leq \kappa_{A, B} .
$$

By the max-flow min-cut theorem [5], a multiflow $f$ locks $\mathcal{A}$ if and only if $f$ attains (2.2) with equality. Therefore the if part and the latter part of Theorem 1.2 are rephrased as follows:

Theorem 2.1. Suppose that $\mathcal{A}$ has no pairwise crossing triple and no irregularly crossing pair. Then, for every nonnegative weight $\alpha: \mathcal{A} \rightarrow \mathbf{R}_{+}$and every network $(G, T, c)$, the following relation holds:

$$
\max \left\{\operatorname{val}_{\mathcal{A}, \alpha}(f) \mid f: \text { multiflow in }(G, T, c)\right\}=\sum_{(A, B) \in \mathcal{A}} \alpha(A, B) \kappa_{A, B}
$$

In addition, if $(G, T, c)$ is properly inner Eulerian, then there exists an integral multiflow $f$ attaining the maximum in (2.3).

It suffices to consider the case where $(G, T, c)$ is properly inner Eulerian. Indeed, for any network $(G, T, c)$ having integer-valued capacity, network $(G, T, 2 c)$ is obviously properly inner Eulerian. Consequently, if (2.3) holds for every properly inner Eulerian network, then it holds for every network having rational-valued capacity. Since both sides of (2.3) are continuous functions on $c$, the relation (2.3) holds for general (irrational) capacity $c$. 
Suppose that $\mathcal{A}$ has no pairwise crossing triple and no irregularly crossing pair and $(G, T, c)$ is property inner Eulerian. Our goal is to prove the existence of an integral multiflow $f$ attaining (2.2) with equality. The proof is based on the splitting-off method. For a simplification of the proof, we use a standard technique to make the input network have a small degree, as in [6, p. 51]. First, by multiplying edges, make each edge have unit capacity. Suppose that there is an inner node $y$ of degree at least 6 , incident to edges $e_{i}=x_{i} y$ $(i=0,1, \ldots, m+1)$ (some nodes $x_{i}, x_{j}$ may coincide). Change the incidence around $y$ by the following way. Subdivide edge $e_{i}=x_{i} y$ into two edges $x_{i} z_{i}, z_{i} y$ for $i=1,2, \ldots, m$. Replace $e_{0}$ by $x_{0} z_{1}$, and replace $e_{m+1}$ by $x_{m+1} z_{m}$. Add new edge $z_{i} z_{i+1}$ for $i=1,2, \ldots, m-1$. Then any integer multiflow in the new network can be transformed into an integer multiflow in the original network having the same $\operatorname{val}_{\mathcal{A}, \alpha}(\cdot)$. The converse transformation is also possible. In particular, the min-cut value $\kappa_{A, B}$ is invariant. By repeating this process, make $y$ have degree four. Suppose that there is an improper terminal $s$ of (even) degree $m \geq 4$. Add $m / 2$ new terminals $s_{1}, s_{2}, \ldots, s_{m / 2}$, and join $s$ and each $s_{i}$ by two parallel edges (of unit capacity). Make $s$ being an inner node, i.e., $T \leftarrow T \backslash\{s\}$. For each partial cut $(A, B) \in \mathcal{A}$ with $s \in A$ (resp. $s \in B$ ), replace $A$ by $A \cup\left\{s_{1}, s_{2}, \ldots, s_{m / 2}\right\} \backslash\{s\}$ (resp. $B$ by $B \cup\left\{s_{1}, s_{2}, \ldots, s_{m / 2}\right\} \backslash\{s\}$ ). Then the problem is unchanged. By this reduction, we may assume that each edge has unit capacity, each inner node has degree four, and each improper terminal has degree two.

Recall the splitting-off operation. A pair $\{x y, y z\}$ of consecutive edges incident to a common node $y$ is called a fork. The splitting-off operation at $\{x y, y z\}$ is to delete edges $x y, y z$ and add a new edge joining $x$ and $z$ of unit capacity (if $x \neq z$ ). A fork is said to be splittable if the splitting-off operation does not decrease the min-cut value $\kappa_{A, B}$ for all $(A, B) \in \mathcal{A}$. The splitting-off decreases the total number of edges. From an integral multiflow in the new network, we obtain an integral multiflow in the original network. Therefore, if we find a splittable fork, then by induction on the number of edges we can prove the existence of an integral multiflow attaining (2.2) in equality.

By the degree condition, each node $x \notin A \cup B$ has even degree for $(A, B) \in \mathcal{A}$. Therefore, for an $(A, B)$-cut $(X, Y), c(X, Y)$ is an even integer if and only if $A$ (or $B$ ) contains the even number of odd-degree vertices. Consequently, for two $(A, B)$-cuts $(X, Y),\left(X^{\prime}, Y^{\prime}\right)$, the difference $c(X, Y)-c\left(X^{\prime}, Y^{\prime}\right)$ is an even integer. Moreover the splitting-off at $\{x y, y z\}$ decreases the cut-capacity of $(X, Y)$ if and only if $y \in X, x, z \in Y$ or $y \in Y, x, z \in X$. If decreases, then it decreases by 2 . Therefore, if $\{x y, y z\}$ is unsplittable, then there exist a partial cut $(A, B) \in \mathcal{A}$ and a minimum $(A, B)$-cut $(X, Y)$ such that $y \in X, x, z \in Y$ or $y \in Y, x, z \in X$. We call this cut $(X, Y)$ a critical $(A, B)$-cut with respect to $\{x y, y z\}$.

Take an inner node $y$ of degree four incident to four edges $e_{i}=y x_{i}$ for $i=0,1,2,3$. We show that at least one of three forks $\left\{x_{0} y, y x_{i}\right\}(i=1,2,3)$ is splittable. Suppose (to the contrary) that all three forks are unsplittable. Then there is a critical $\left(A_{i}, B_{i}\right)$-cut $\left(X_{i}, Y_{i}\right)$ with respect to $\left\{x_{0} y, y x_{i}\right\}$ for $i=1,2,3$. We claim:

(*1) $\left(A_{i}, B_{i}\right)$ and $\left(A_{j}, B_{j}\right)$ are crossing if $i \neq j$.

This immediately leads a contradiction to the hypothesis of $\mathcal{A}$. Suppose that $\left(A_{1}, B_{1}\right)$ and $\left(A_{2}, B_{2}\right)$ are laminar. We may assume $x_{0}, x_{i} \in X_{i}$ and $y \in Y_{i}$ for $i=1,2$. Then $x_{2}, x_{3} \in Y_{1}$ and $x_{1}, x_{3} \in Y_{2}$ necessarily hold. Indeed, if $x_{2} \in X_{1}$, then $c\left(X_{1}, Y_{1}\right)>c\left(X_{1} \cup\{y\}, Y_{1} \backslash\{y\}\right)$; this is a contradiction to the assumption that $\left(X_{1}, Y_{1}\right)$ is a minimum $\left(A_{1}, B_{1}\right)$-cut. It suffices to consider two cases: (i) $A_{1} \subseteq A_{2}, B_{1} \supseteq B_{2}$ and (ii) $A_{1} \subseteq B_{2}, B_{1} \supseteq A_{2}$. Suppose (i). Recall the submodular-type relation of cuts:

$$
c\left(X_{1}, Y_{1}\right)+c\left(X_{2}, Y_{2}\right)=c\left(X_{1} \cap X_{2}, Y_{1} \cup Y_{2}\right)+c\left(X_{1} \cup X_{2}, Y_{1} \cap Y_{2}\right)+2 c\left(X_{1} \backslash X_{2}, X_{2} \backslash X_{1}\right)
$$


Then $\left(X_{1} \cap X_{2}, Y_{1} \cup Y_{2}\right)$ is a minimum $\left(A_{1}, B_{1}\right)$-cut and $\left(X_{1} \cup X_{2}, Y_{1} \cap Y_{2}\right)$ is a minimum $\left(A_{2}, B_{2}\right)$-cut. However $c\left(X_{1} \cup X_{2} \cup\{y\}, Y_{1} \cap Y_{2} \backslash\{y\}\right)<c\left(X_{1} \cup X_{2}, Y_{1} \cap Y_{2}\right)$. A contradiction to the minimality. So suppose (ii). Then $\left(X_{1} \cap Y_{2}, Y_{1} \cup X_{2}\right)$ and $\left(X_{1} \cup Y_{2}, Y_{1} \cap X_{2}\right)$ are an $\left(A_{1}, B_{1}\right)$-cut and an $\left(A_{2}, B_{2}\right)$-cut, respectively. There is one more relation:

$$
c\left(X_{1}, Y_{1}\right)+c\left(X_{2}, Y_{2}\right)=c\left(X_{1} \cap Y_{2}, Y_{1} \cup X_{2}\right)+c\left(X_{1} \cup Y_{2}, Y_{1} \cap X_{2}\right)+2 c\left(X_{1} \cap X_{2}, Y_{1} \cap Y_{2}\right) \text {. }
$$

By $x_{0} \in X_{1} \cap X_{2}, y \in Y_{1} \cap Y_{2}$, and $e_{0}=x_{0} y \in E G$. we have $c\left(X_{1} \cap X_{2}, Y_{1} \cap Y_{2}\right)>0$. So $c\left(X_{1}, Y_{1}\right)>c\left(X_{1} \cap Y_{2}, Y_{1} \cup X_{2}\right)$ or $c\left(X_{2}, Y_{2}\right)>c\left(X_{1} \cup Y_{2}, Y_{1} \cap X_{2}\right)$. However this contradicts to the minimality assumption. Therefore every inner node has a splittable fork.

So it suffices to consider the case where there is no inner node, i.e., $V G=T$. Suppose further that a (unique) fork at every improper terminal is unsplittable; if splittable, then split it off and apply induction. We claim:

(*2) for each $(A, B) \in \mathcal{A}$ we have $c(A, B)=\kappa_{A, B}$.

If true, then the set of all one-edge paths of unit flow-value is obviously an integral multiflow attaining (2.2) with equality. So suppose $c(A, B)<\kappa_{A, B}$. By Menger's theorem, there is a path $\left(s_{0}, s_{1}, \ldots, s_{m}\right)$ such that $s_{0} \in A, s_{m} \in B$, and $s_{j} \notin A \cup B$ for $j=1,2, \ldots, m-1$ $(m \geq 2)$. For $j=1,2, \ldots, m-1$, terminal $s_{j}$ is improper and is incident only to $s_{j-1}$ and $s_{j+1}$. Consider the splitting-off at a fork $\left\{s_{0} s_{1}, s_{1} s_{2}\right\}$. By assumption, it is unsplittable. Take its critical $\left(A^{\prime}, B^{\prime}\right)$-cut $(X, Y)$ with $s_{1} \in X$ and $s_{0}, s_{2} \in Y$. Then necessarily $s_{1} \in A^{\prime}$; otherwise $\left(X \backslash\left\{s_{1}\right\}, Y \cup\left\{s_{1}\right\}\right)$ is an $\left(A^{\prime}, B^{\prime}\right)$-cut having smaller cut capacity. Thus $A^{\prime} \cup B^{\prime} \neq A \cup B$, and consequently $\left(A^{\prime}, B^{\prime}\right),(A, B)$ are laminar. By laminarity we have $s_{m} \in B \subseteq A^{\prime} \subseteq X$ (and $\left.A \supseteq B^{\prime}\right)$. Then $s_{2} \in B(m=2)$ is impossible. So $m>2$. However we have $c(X, Y)>c\left(X \cup\left\{s_{2}, s_{3}, \ldots, s_{m-1}\right\}, Y \backslash\left\{s_{2}, s_{3}, \ldots, s_{m-1}\right\}\right)$; a contradiction to the minimality of $(X, Y)$.

\section{Concluding Remarks}

The size of lockable family. Karzanov and Lomonosov [14] asked: how large is the size of a lockable family? A set system without crossing triple is said to be 3-cross-free. Pevzner [17] showed that the cardinality of any 3-cross-free family on $n$-set is $O(n)$; see [4] for a shorter proof. Recently, Dress, Koolen and Moulton [3] proved the tight upper bound $8 n-20(n \geq 3)$.

A similar question arises: how large is the size of a lockable family of partial cuts ? We show that the size of a lockable family of partial cuts on $n$-set is not linearly bounded, but is bounded by $O\left(n^{2}\right)$. We give an example of $O\left(n^{2}\right)$ size. Let $n=2 k$ be a positive even integer. Consider a tree $\Gamma$ consisting of edges $x_{0} x_{1}^{i}, x_{1}^{i} x_{2}^{i}, \ldots, x_{k-1}^{i} x_{k}^{i}$ for $i=1,2, \ldots, k$. Namely $\Gamma$ is obtained by subdividing a star of $k$ leaves. Let $[n]=\{1,2, \ldots, n\}$ be an $n$-element set. We associate each element $j$ in $[n]$ with a subtree $F_{j}$ in $\Gamma$ as follows. For $j=1,2, \ldots, k$, let $F_{j}$ be the subtree induced by nodes $x$ with $d_{\Gamma}\left(x_{0}, x\right) \leq j-1$, where $d_{\Gamma}$ is the shortest path metric on node set of $\Gamma$. For $j=k+1, k+2, \ldots, 2 k$, let $F_{j}$ be the subtree consisting of one node $x_{k}^{j-k}$. For each edge $e$ in $\Gamma$, the deletion of $e$ divides $\Gamma$ into two connected components $\Gamma^{\prime}, \Gamma^{\prime \prime}$. Let $A_{e}$ be the set of indices $i$ for which $F_{i}$ belongs to $\Gamma^{\prime}$, and let $B_{e}$ be the set of indices $i$ for which $F_{i}$ belongs to $\Gamma^{\prime \prime}$. Note that if $F_{j}$ contains $e$, then neither $A_{e}$ nor $B_{e}$ contains $j$. Now we obtain a partial cut family $\mathcal{A}=\left\{\left(A_{e}, B_{e}\right) \mid e\right.$ is an edge of $\left.\Gamma\right\}$ of cardinality $(n / 2)^{2}=O\left(n^{2}\right)$. One can easily verify that $\mathcal{A}$ is laminar, i.e., each pair is laminar. In fact, it is known that every laminar partial cut family is obtained by a family of subtrees on a tree in this way [9]. 
To see the upper bound $O\left(n^{2}\right)$, we use a result from the split decomposition theory $[1,8]$; a partial cut is called a partial split in [8, Section 4]. Then one can easily see that a set of partial cuts without crossing triple and irregularly crossing pair is weakly compatible in the sense of [8, Section 4]. A general result [8, Theorem 4.13] says that for a weakly compatible family of partial cuts $\mathcal{A}$ on an $n$-set $T$, the corresponding set of cut distances $\left\{\delta_{A, B} \mid(A, B) \in\right.$ $\mathcal{A}\}$ is linearly independent in the vector space of symmetric functions with zero diagonals $\left\{d \in \mathbf{R}^{T \times T} \mid d(s, t)=d(t, s), d(s, s)=0(s, t \in T)\right\}$. Therefore $|\mathcal{A}| \leq n(n-1) / 2=O\left(n^{2}\right)$.

Fractionality. Theorem 2.1 says the existence of an integral optimal multiflow in a class of $\mu$-weighted maximum multiflow problems. For a weight $\mu: T \times T \rightarrow \mathbf{R}_{+}$, the fractionality of $\mu$ is defined to be the least positive integer $k$ with property that the $\mu$-weighted maximum multiflow problem has a $1 / k$-integral optimal multiflow for every integer-capacitated network $(G, T, c)$. If such an integer does not exist, then the fractionality is defined to be infinity. Recently, [11] proved a complete characterization of weights having finite fractionality, and showed that if the fractionality is finite, then it is a divisor of 24 (the conjectured tight upper bound is 4). So it is still interesting to identify a class of weights having small fractionality. From the point of the view, Theorem 2.1 provides a new class of weights having fractionality 2. A distance among subtrees in a tree, considered in [9], is a natural example of such a weight; it is exactly a nonnegative sum of cut distances for a laminar partial cut family. By the extended split decomposition in [8, Section 4], we can determine, in strongly polynomial time, whether a given weight $\mu$ is decomposed into a nonnegative sum of cut distances for a lockable family of partial cuts, and we can also obtain an explicit decomposition if decomposable.

A polynomial time algorithm. The splitting-off proof provides a strongly polynomial time algorithm to find an integral multiflow locking $\mathcal{A}$ in a properly inner Eulerian network. We sketch it. Once the existence of an integral solution is established, it is unnecessary to reduce the degrees as in the proof of Theorem 2.1. So we may assume that the input network $(G, T, c)$ is complete and has no multiple edges and loops. Let $n=|V G|$. We use a capacitated version of splitting-off. The maximum capacity of the splitting-off can be computed, in strongly polynomial time, by a minimum $(A, B)$-cut algorithm for all $(A, B) \in$ $\mathcal{A}$. By applying splitting-off for all $n^{2}(n+1) / 2$ forks (in some ordering), we can make the network have no splittable fork. This is nontrivial; see [11, Section 7] and [13, Section 4] for details. Then the multiflow consisting of all one-edge paths is obviously an integral solution. By reversing the splitting-off operations, we obtain an integral multiflow locking $\mathcal{A}$ in the original network (in an edge-node form if necessarily). So the whole complexity is $O\left(n^{3}|\mathcal{A}| \varphi(n)\right)$, where $\varphi(n)$ denotes the complexity of a maximum flow algorithm for an $n$-node network. An augmenting path approach, as in $[2,12,15]$, would yield a more faster algorithm, which is left to readers.

\section{Acknowledgements}

The author thanks the referees for helpful comments. This work is supported by a Grantin-Aid for Scientific Research from the Ministry of Education, Culture, Sports, Science and Technology of Japan. 


\section{References}

[1] H.-J. Bandelt and A. Dress: A canonical decomposition theory for metrics on a finite set. Advances in Mathematics, 92 (1992), 47-105.

[2] B.V. Cherkassky: A solution of a problem of multicommodity flows in a network. Ekonomika i Matematicheskie Metody, 13 (1977), 143-151 (in Russian).

[3] A.W.M. Dress, J. Koolen, and V. Moulton: 4n-10. Annals of Combinatorics, 8 (2004), 463-471.

[4] T. Fleiner: The size of 3-cross-free families. Combinatorica, 21 (2001), 445-448.

[5] L.R. Ford, Jr. and D.R. Fulkerson: Flows in Networks (Princeton University Press, Princeton, 1962).

[6] A. Frank: Packing paths, circuits, and cuts — a survey. In B. Korte, L. Lovász, H. J. Prömel, and A. Schrijver (eds.): Paths, Flows, and VLSI-Layout (Springer, Berlin, 1990), 47-100.

[7] A. Frank, A.V. Karzanov, A. Sebö: On integer multiflow maximization. SIAM Journal on Discrete Mathematics, 10 (1997), 158-170.

[8] H. Hirai: A geometric study of the split decomposition. Discrete and Computational Geometry, 36 (2006), 331-361.

[9] H. Hirai: Characterization of the distance between subtrees of a tree by the associated tight span. Annals of Combinatorics, 10 (2006), 111-128.

[10] H. Hirai: Folder complexes and multiflow combinatorial dualities. RIMS-Preprint 1675, 2009.

[11] H. Hirai: The maximum multiflow problems with bounded fractionality. RIMS-Preprint 1682, 2009.

[12] T. Ibaraki, A.V. Karzanov, and H. Nagamochi: A fast algorithm for finding a maximum free multiflow in an inner Eulerian network and some generalizations. Combinatorica, 18 (1998), 61-83.

[13] A.V. Karzanov: On a class of maximum multicommodity flow problems with integer optimal solutions. In A.K. Kelmans (ed.): Selected Topics in Discrete Mathematics (American Mathematical Society, Providence, 1994), 81-99.

[14] A.V. Karzanov and M.V. Lomonosov: Flow systems in undirected networks. In O.I. Larichev (ed.): Mathematical Programming (Institute for System Studies, Moscow, 1978), 59-66 (in Russian).

[15] M.V. Lomonosov: Combinatorial approaches to multiflow problems. Discrete Applied Mathematics, 11 (1985), 1-93.

[16] L. Lovász: On some connectivity properties of Eulerian graphs. Acta Mathematica Academiae Scientiarum Hungaricae, 28 (1976), 129-138.

[17] P.A. Pevzner: Non-3-crossing families and multicommodity flows. In A.K. Kelmans (ed.): Selected Topics in Discrete Mathematics (American Mathematical Society, Providence, 1994), 201-206.

[18] A. Schrijver: Combinatorial Optimization-Polyhedra and Efficiency (Springer-Verlag, Berlin, 2003).

\section{Hiroshi Hirai}

Research Institute for Mathematical Sciences, Kyoto University, 
Kyoto 606-8502 Japan

E-mail: hirai@kurims.kyoto-u.ac.jp 